${ }^{8}$ LiU, SHI-JIE (1985) An outline of the grass-roots health care system in the People's Republic of China. In Mental Health Planning for One Billion People: A Chinese Perspective. (Eds Lin, T-Y and L. Eisenberg). University of British Columbia Press.
${ }^{9}$ Peng, Rui-Cong (1985) A biopsychosocial medical model and medical education. In Mental Health Planning for One Billion People. (Eds Lin, T-Y and L. Eisenberg) Unversity of British Columbia Press.

\title{
Report from the People's Republic of China
}

\section{J. H. Henderson, Medical Director, St Andrew's Hospital, Northampton}

An international workshop on Epidemiology of Mental and Neurological Disorders of the Elderly was held in the Medical University of Beijing in the People's Republic of China from 16-20 November 1987. The workshop was organised jointly by the Beijing Institute of Mental Health and the WHO Collaborating Centre for Research and Training in the Neurosciences, Milan, Italy.

With sponsorship from the Division of Neurosciences of the International Foundation Fatebenefratelli, Milan, Italy, a faculty of nine international experts coming mainly from WHO Collaborating Centres for Research and Training in Mental Health, Neurosciences and Health of the Elderly, presented their experience of recent trends from various branches of clinical and scientific medicine and exchanged technical information with about one hundred clinicians, scientists and researchers, representing every region of the People's Republic of China.

As in many countries throughout the world, mental health care in China is in a state of transition from that of a traditional mental hospital-based service towards a specialist service of community-based facilities, centred on the mental hospital or sizeable unit for psychiatry attached to a general hospital. ${ }^{1}$ These patterns of change in mental health care have been apparent for more than a decade now and progress has been considerable and rapid throughout the land. ${ }^{2}$

The Cultural Revolution, 1966-1976, wreaked untold havoc in the country's medical and educational systems; and the neurosciences and psychiatry in particular were severely curtailed and denigrated. Since 1976, however, there has been a significant resurgence of education, research and development in the neurosciences and in psychiatry. ${ }^{1,2}$
Both Parry-Jones and Crammer in recent accounts of visits to psychiatric services in the People's Republic of China, whilst mentioning organic states among the clinical problems present in the Chinese population, make no mention specifically of the psychiatric problems of old age. ${ }^{1,2}$ China, with its huge population in excess of one billion people, is already aware of the increasing demand made on health services from the elderly with mental illness, and particularly those with dementia.

The demographic changes taking place in the country are significant, while the socio-cultural changes in family structure are no less so. That the prevalence of dementia in the population will continue to increase and pose considerable problems for the psychiatric and medical services cannot be in doubt. Life expectancy has increased from 35 years in 1940-before the foundation of the People's Republic of China - to nearly 70 years today. In the capital city, Beijing, $15 \%$ of the population will be over 65 years of age by the year 2000 . Whilst the country's Constitution states that adult sons and daughters are obliged to take care of their parents, it is a fact that already in 1987 more than $20 \%$ of persons aged 60 years and over in Beijing lived alone and away from their children, while less than $3 \%$ of persons aged 60 and over in rural areas lived alone and away from their children. In the rural areas the elderly are still virtually all cared for by their descendants and virtually none enter old peoples' homes.

Early in 1986 the Institute of Mental Health in Beijing undertook an epidemiological survey in an urban district of the metropolis to determine the prevalence of dementia in the elderly population. A rigorous protocol was followed, and a prevalence rate for moderate and severe dementia in the population aged 65 and over was $1.82 \% .^{3}$ When 
age-adjusted for comparison with prevalence data from the USA, the prevalence was estimated at $3.2 \%$. The data did show, too, a prevalence rate increasing rapidly with age, a tendency already reported from the USA and Europe. ${ }^{4.5}$

Of striking significance in this survey, as reported to the visiting scientists, was the finding that cases of multi-infarct dementia were found more often than cases of dementia of Alzheimer's type. This is consistent with similar findings from Japan but clearly at odds with findings in the USA and Europe, where in similar community studies cases of dementia of Alzheimer's type are consistently significantly greater in number than cases of multi-infarct dementia. ${ }^{6}$

Also of great interest to the scientists from the USA and Europe was the prominent finding that in this community study every one of the cases of dementia detected was being taken care of in his/her own home or in the homes of family and near relatives. None of them had been seen by a doctor; none of them had ever been admitted to a hospital for any mental disorder and, significantly, the relatives considered no need for medical or social interventions since the evident cognitive decline was considered to be a natural consequence of the ageing process.

This latter finding alone has been sufficient justification and encouragement for the Institute of Mental Health in Beijing to take the initiative to promote this national workshop on mental and neurological disorders of the elderly.
The doctors and nurses and those concerned with the problems of an ageing population must extend their range of knowledge and awareness of the importance of diagnosis, classification, treatment and the provision of adequate care of the elderly with mental neurological disorders. Mental health care for the elderly must inevitably become an important component of this country's national plan for mental health.

\section{References}

${ }^{1}$ Parry-Jones, W. Ll. (1986) Psychiatry in the People's Republic of China. British Journal of Psychiatry, 148, 632-641.

${ }^{2}$ Crammer, J. L. (1985) Two weeks work in SW China. Bulletin of the Royal College of Psychiatrists, 3, 56-58.

${ }^{3}$ LI, G., ShEN, Y. C., CHEN, C. H. et al (1986) An epidemiological survey of age-related dementia in an urban area of Beijing, Institute of Mental Health, Beijing, People's Republic of China.

${ }^{4}$ Henderson, A. S. (1986) The epidemiology of Alzheimer's disease. British Medical Bulletin, 42, 3-10.

${ }^{5}$ MorTimer, J. A. et al (1981) Epidemiology of dementing illness. In The Epidemiology of Dementia (Eds. J. A. Mortimer \& L. M. Schuman). New York: Oxford University Press.

${ }^{6}$ Henderson, A. S. In Etiology of Dementia of Alzheimer's type. (Eds. A. S. Henderson \& J. H. Henderson). Chichester: John Wiley. p 198 (in press). 